\title{
Évolution et rôle des réserves glucidiques et azotées des tiges chez 21 génotypes de blé
}

\author{
E Triboi, JL Ollier \\ avec la collaboration technique de D Ezard \\ INRA, station de recherches agronomiques, 63039 Clermont-Ferrand, France ${ }_{\boldsymbol{\theta}}$ \\ (Reçu le 14 février 1990; accepté le 22 février 1991)

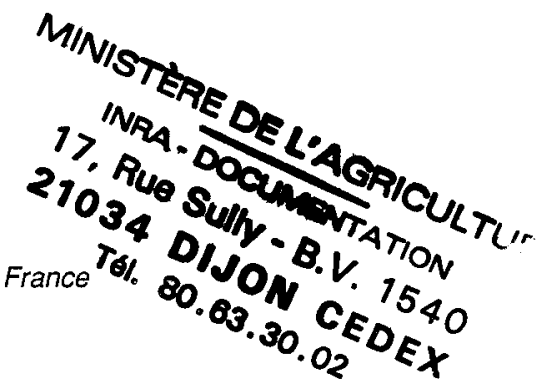

\begin{abstract}
Résumé - L'évaluation et le rôle des réserves glucidiques et azotées des tiges ont été analysés sur 21 génotypes de blé cultivés au champ. Les teneurs moyennes en glucides solubles et $N$ total ont été respectivement de $15,4 \%$ et $1,1 \%$ à la floraison et $2,8 \%$ et $0,5 \%$ à la récolte. Les réserves glucidiques augmentent pendant environ $10 \mathrm{j}$ après floraison et diminuent ensuite de $258 \mathrm{mg} .{ }^{\circ} \mathrm{C}^{-1} \cdot \mathrm{j}^{-1} \cdot \mathrm{m}^{-2}$. Le flux du carbone exporté depuis la tige $\left(\mathrm{mg}^{\circ} \mathrm{C}^{-1} \mathrm{j}^{-1} \cdot \mathrm{m}^{-2}\right)$ est corrélé positivement avec le niveau maximal des réserves glucidiques mesurées $10 \mathrm{j}$ après floraison. Une variation de $10 \mathrm{~g} \cdot \mathrm{m}^{-2}$ de réserves correspond à une variation du flux exporté de $15 \mathrm{mg} .{ }^{\circ} \mathrm{C}^{-1} \mathrm{j}^{-1} \cdot \mathrm{m}^{-2}$. La contribution des réserves $\mathrm{C}$ au remplissage des grains a été d'environ $15 \%$. Les réserves $\mathrm{N}$ sont stables pendant environ $10 \mathrm{j}$ après floraison et diminuent ensuite avec un flux de $6,7 \mathrm{mg} .{ }^{\circ} \mathrm{C}^{-1} \mathrm{j}^{-1} \mathrm{~m}-2$. L'analyse des composantes du rendement a mis en évidence un effet favorable du nombre de grains par $\mathrm{m}^{2}$ (NG) et du rendement par épi (RDTE) sur le rendement (RDT). Selon la stratégie utilisée dans la production d'un NG donné, en favorisant le nombre d'épis ou la fertilité de l'épi, la quantité des réserves par tige varie. En conditions difficiles de milieu pendant la période de remplissage des grains, il semblerait qu'une structure de peuplement à nombre d'épis faible mais à fertilité élevée, accompagnée par des réserves importantes dans la tige, soit favorable. La vitesse de remplissage augmente, la durée diminue et le risque d'intervention des facteurs limitants est plus faible.
\end{abstract}

réserve / transfert / composante du rendement $/$ génotype $/$ Triticum $=$ blé

Summary - Kinetic and role of $\mathbf{C}$ and $\mathbf{N}$ stored in stem on 21 wheat genotypes. The dynamics and the role of water-soluble carbohydrate (WSC) and nitrogen (N) stored in the stem since anthesis were analysed over 21 genotypes. The yield components and the rate and duration of grain filling characterizing the 21 genotypes are presented in table II. The mean contents of WSC and N were 15.4 and $1.1 \%$ at flowering (table I) and $2.8 \%$ and $0.5 \%$ at harvest time (fig 1). The WSC continued to increase for $\approx 10 d$ after anthesis (fig 1a); thereafter, WSC in stems decreased by $258 \mathrm{mg} .{ }^{\circ} \mathrm{C}^{-1} . \mathrm{d}^{-1} . \mathrm{m}^{2}$ (fig 2a). The $C$ flux exported from the stem was correlated with the level of stem reserve (C) analysed $10 \mathrm{~d}$ after anthesis (fig 3). It is also possible that the biochemical composition of WSC changes the $C$-flux from the stem (table IV). The stem WSC contribution to yield was $\approx 15 \%$ (table III). The amount of $N$ stored into the stem remained stable for $10 \mathrm{~d}$ after flowering; thereafter $\mathrm{N}$ in the stem decreased by $6.7 \mathrm{mg} .{ }^{\circ} \mathrm{C}^{-1} . \mathrm{d}^{-1} . \mathrm{m}^{2}$ (fig $2 \mathrm{~b}$ ). The yield component analysis showed a favourable effect of number of kernels $/ \mathrm{m}^{2}$ (NG) joint to yield per ear (fig 4). In consequence, the strategy of NG production, based on ear fertility or on ear density, has an effect on the stem reserves. In unfavorable environments it is possible that crop yield structure based on a low number of ear/m² but with a high degree of fertility will be more interesting (table $\mathrm{V}$ ). The rate of grain filling increases and the duration decreases; consequently the risk of intervention of unfavorable factors is decreased.

stem reserves / trans/ocation / yield components / genotype / Triticum = wheat

\section{INTRODUCTION}

Chez le blé, l'étude des réserves carbonées et azotées présente un intérêt particulier pendant la période post-floraison car ces réserves contribuent à l'élaboration du poids d'un grain et de sa qualité. En effet, le flux d'assimilats $\mathrm{C}$ et $\mathrm{N}$ vers le grain est constitué d'une part par des assimilats récents provenant de l'assimilation nette postfloraison du $\mathrm{CO}_{2}$ et de l'absorption racinaire de l'azote et d'autre part par des assimilats provenant de la mobilisation des réserves, stockées principalement dans les tiges (Simmons, 1987). La connaissance de la contribution de chacune de ces 2 sources est nécessaire pour modéliser 
le fonctionnement d'un peuplement de blé en vue de maîtriser l'élaboration du poids d'un grain.

La contribution globale des réserves au rendement final est aujourd'hui assez bien connue. Ainsi, la contribution des réserves carbonées est variable, de 5 à plus de $30 \%$, selon la quantité de $\mathrm{CO}_{2}$ assimilé après la floraison, modifiée essentiellement par les conditions climatiques et celle des réserves (Austin et al, 1980; Blacklow et al, 1984; Triboi et al, 1985b; Kuhbauch et Thome, 1989). En ce qui concerne les réserves azotées, la part de l'azote absorbé avant floraison est nettement plus importante, car seulement $30 \%$ environ du $\mathrm{N}$ présent dans le grain à la maturité provient de l'absorption post-floraison (Austin et al, 1977).

En revanche, l'évolution saisonnière des réserves, même si elle a été mise en évidence dans des nombreuses études (Pollock et Jones, 1979; Judel et Mengel, 1982; Blacklow et al, 1984; Kuhbauch et Thome, 1989) n'a pas été souvent quantifiée en terme de flux d'entrée et de sortie (Simpson et al, 1983). Les effets des différents facteurs sur la constitution, l'utilisation et le rôle stratégique des réserves dans l'élaboration du rendement et dans l'adaptation au milieu, et notamment les effets dus au génotype, restent à préciser.

Dans cet article, nous présentons les résultats obtenus en expérimentant 21 génotypes de blé d'hiver.

\section{MATÉRIEL ET MÉTHODES}

Vingt et une variétés de blé d'hiver ont été cultivées dans un sol argilo-calcaire de Limagne, en utilisant les techniques de production intensive employées dans cette zone céréalière. Les variétés expérimentées, ordonnées selon la date de floraison ont été : Talent, Garant, Gerbier, Festival, Feuvert, Courtel, Arsenal, Tarasque, Thésée, Chopin, Fidel, H10, Aristide, Promontin, Pernel, Belaviso, C Rémy, Epiroux, Moulin, Master, Arminda.

Des échantillons de plantes ont été prélevés sur 3 répétitions de 2 lignes contiguës de $1 \mathrm{~m}$, à 5 dates : à la floraison, à environ 11,17 et $25 \mathrm{j}$ après floraison et à la récolte. Après la constitution d'un échantillon moyen de 50-100 tiges portant un épi, les différents organes (feuilles, épi, tige) ont été séparés. Les tiges et les graines enveloppantes ont été coupées en morceaux d'environ $1 \mathrm{~cm}$. Une partie a été mise à l'étuve $48 \mathrm{~h}$ à $90^{\circ} \mathrm{C}$ pour déterminer l'humidité et la teneur en $\mathrm{N}$ total; une autre partie, d'environ $10 \mathrm{~g}$ a été stockée à $-30^{\circ} \mathrm{C}$ et utilisée pour le dosage des glucides.
Les fructosanes représentant le composant majeur des carbohydrates non structuraux mis en réserve (Judel et Mengel, 1982; Blacklow et al, 1984), nous n'avons analysé que les glucides solubles sans tenir compte de l'amidon présent en quantité insignifiante (Kuhbauch et Thome, 1989).

Deux extractions successives de 45-30 min ont été effectuées à $85^{\circ} \mathrm{C}$ par une solution aqueuse à $40 \%$ d'éthanol. Après rinçage, purification par le charbon actif et ultrafiltration, le dosage des glucides a été effectué par la méthode à l'anthrone sulfurique, référence glucose. De plus, les proportions des différentes formes glucidiques (glucose, fluctose, saccharose, etc) ont été déterminées par HPLC sur les échantillons présentant les plus faibles et les plus fortes teneurs, en utilisant comme phase stationnaire la résine Biorad XPX 87P et comme phase mobile, l'eau ultrapure. L'identification et l'évolution de différentes formes glucidiques ne seront pas abordées ici.

Les composantes du rendement, nombre d'épis $/ \mathrm{m}^{2}$ (NE) et nombre de grains par épi (NGE) ont été déterminées sur les 4 derniers prélèvements et le poids d'un grain $(p)$ sur le prélèvement à la récolte. Le poids d'un grain est assimilé au produit de la vitesse de remplissage $(v)$ par la durée de remplissage $(D),(p=v x$ $D$ ). Dans ce cas la vitesse "v» est calculée par régression dans la période de croissance linéaire du grain (Triboi et al, 1985a, 1985b; Triboi, 1990).

\section{RÉSULTATS}

\section{Niveaux des réserves glucidiques (RG) et azotées $(R N)$ à la floraison}

La teneur moyenne en glucides solubles établie sur 21 génotypes est de $15,4 \%$ du poids sec de tige entière (tableau I).

Le rapport des teneurs extrêmes est de 2, si elles sont exprimées en pourcentage du poids sec, 2,8 si elles le sont en mg/tige et 1,8 si elles le sont en $\mathrm{g} / \mathrm{m}^{2}$. Pour l'azote, la teneur moyenne est de $1,1 \%$ du poids sec de tige avec des rapports maximaux de $2,1,8$ et 1,6 selon que l'on s'exprime en concentration, en $\mathrm{mg} /$ tige et en $\mathrm{g} /$ $\mathrm{m}^{2}$. Bien que dans cet essai, le génotype accumulant le moins de réserves glucidiques (Arminda) soit celui qui contienne la plus forte quantité de protéines/tige, à ce stade de prélèvement aucune corrélation n'existe entre niveaux $\mathrm{C}$ et $\mathrm{N}$.

La quantité de glucides par tige à la floraison est corrélée au nombre de tiges par $\mathrm{m}^{2}$ portant épi, égal donc au nombre d'épis par $\mathrm{m}^{2}$ (NE) :

$R G /$ tige : $-0,188 N E+292 ; n=21 ; r=-0,67$.

Exprimées par $\mathrm{m}^{2}$, ces réserves carbonées sont corrélées à la date de floraison $D F(D F$ en j, avec $D F=1$ le 1 er janvier 1986) :

$$
R G / \mathrm{m}^{2}=3,80 D F-534 ; n=19 ; r=0,74
$$


Tableau I. Caractéristiques moyennes à la floraison des 21 génotypes.

\begin{tabular}{|c|c|c|c|c|c|c|c|c|c|c|}
\hline & \multicolumn{3}{|c|}{$\begin{array}{l}\text { Glucides } \\
\text { réf glucose }\end{array}$} & \multicolumn{3}{|c|}{ Azote total } & \multicolumn{4}{|c|}{$\begin{array}{l}\text { Caractéristiques } \\
\text { du peuplement }\end{array}$} \\
\hline & $\%$ & mg/tige & $g / m^{2}$ & $\%$ & mg/tige & $g / m^{2}$ & mg/tige & $N E / m^{2}$ & dateDF & MSTmg \\
\hline Moyenne & 15,4 & 140,0 & 96,7 & 1,10 & 9,9 & 6,9 & 915 & 703 & $16 / 6$ & 1251 \\
\hline Erreur sdt & 0,6 & 6,9 & 4,0 & 0,04 & 0,3 & 0,2 & 31 & 25 & 1 & 34 \\
\hline Minimum & 10,1 & 77,7 & 65,3 & 0,76 & 7,1 & 5,5 & 653 & 467 & $10 / 6$ & 982 \\
\hline Maximum & 21 & 217,9 & 119,8 & 1,49 & 12,3 & 8,7 & 1185 & 818 & $24 / 6$ & 1580 \\
\hline
\end{tabular}

Les quantités de réserves azotées par tige sont également corrélées au nombre de tiges $/ \mathrm{m}^{2}$ $(r=0,66 ; n=21)$ et à la date de floraison $(r=$ $0,54 ; n=19)$.

\section{Dynamique des réserves $C$ et $N$ entre floraison et récolte}

Les évolutions des niveaux $\mathrm{C}$ et $\mathrm{N}$ entre la floraison et la date du dernier prélèvement effectué après la fin de la période de remplissage du grain, d'une durée moyenne de $43 \pm 1$ j ou de $882 \pm 15$ degrés.jour $\left({ }^{\circ} \mathrm{C} . j\right)$, ne sont pas identiques.

Les concentrations en azote décroissent constamment jusqu'à la récolte où elles ne réprésen-
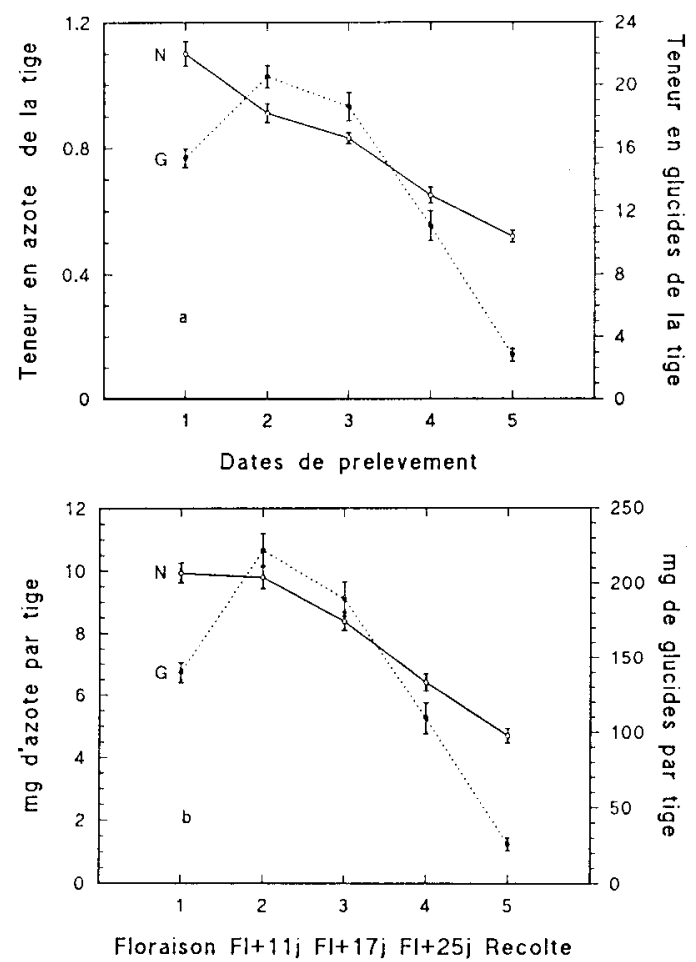

Fig 1. Évolution des réserves glucidiques $(G)$ et azotées $(N)$ dans les tiges de blé. a: exprimées en concentration; b: exprimées en quantités par tige. tent plus que $0,5 \%$ (fig 1a). Par contre, celles en glucides solubles augmentent et atteignent un maximum de $20,5 \%$ du poids sec de tige $11 \mathrm{j}$ après la floraison (selon nos dates de prélèvement), puis diminuent jusqu'à la récolte, stade auquel elles représentent encore $2,8 \%$ de la matière sèche. Exprimées en $\mathrm{mg} /$ tige, les réserves azotées sont stables au cours des 11 j suivant la floraison puis diminuent jusqu'à la récolte (fig 1b). Les réserves carbonnées présentent la même évolution que lorsqu'elles sont exprimées en concentration.

Les vitesses moyennes de transfert à partir des tiges pendant la période comprise entre floraison $+11 \mathrm{j}$ et récolte, évaluée en moyenne à $658 \pm 18{ }^{\circ} \mathrm{C} . j$, sont de $258 \pm 23 \mathrm{mg} .{ }^{\circ} \mathrm{C} . \mathrm{j}^{-1} \cdot \mathrm{m}^{-2}$ pour les glucides solubles (fig 2 a) et de $6,7 \pm$
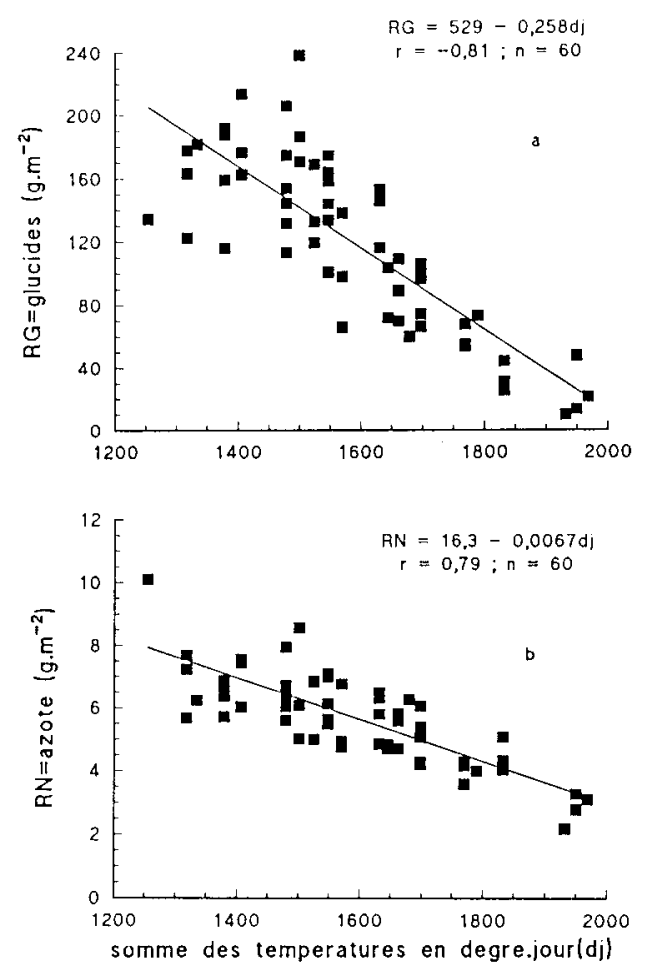

Fig 2. Décroissance des réserves des tiges au cours de la croissance du grain. a: réserves glucidiques (RG); b: ré serves azotées (RN). 
$0,65 \mathrm{mg} .{ }^{\circ} \mathrm{C} . \mathrm{j}^{-1} \cdot \mathrm{m}^{-2}$ pour l'azote (fig 2b) . Pour les glucides, une forte corrélation apparaît entre vitesse de ces transferts et quantité de réserves $/ \mathrm{m}^{2}(r=0,88 ; n=20$, fig 3$)$. Les génotypes à fortes réserves en $C$ possèdent aussi une vitesse élevée d'utilisation de ces réserves. Ce résultat n'existe pas avec l'azote.

\section{Les composantes du rendement}

Les valeurs moyennes des composantes du rendement pour les 21 variétés sont présentées dans le tableau II. Elles reflètent un bon potentiel de production car le rendement moyen a été $69,4 \mathrm{q} /$ ha de matière sèche.

L'étude des relations entre les différentes composantes du rendement, présentée dans la figure 4, confirme le modèle général élaboré antérieurement (Triboi, 1986). Notons que dans cette expérimentation, le nombre de grains par $\mathrm{m}^{2}$ est plus fortement corrélé au nombre de grains par épi qu'au nombre d'épis par $\mathrm{m}^{2}$. En effet, pour l'année de l'expérimentation, la fertilité de l'épi a été contrôlée en grande partie par les conditions climatiques pendant la méiose.

Le poids d'un grain est assez bien corrélé à la vitesse de remplissage du grain $(r=0,58 ; n=$ 21).

Un nombre de grain par $m^{2}$ élevé, bien qu'il diminue le flux d'assimilats par grain $(v)(r=$ $-0,52)$, augmente le flux par $\mathrm{m}^{2}(V),(r=0,62)$.

Finalement, le rendement (RDT) a été fortement corrélé au nombre de grains $N G(r=0,73)$. Aucune relation n'est mise en évidence avec le poids du grain. L'influence de cette composante est cependant retrouvée à travers la variable rendement par épi (RDTE). Celle-ci représente en effet une variable centrale car elle est corré-

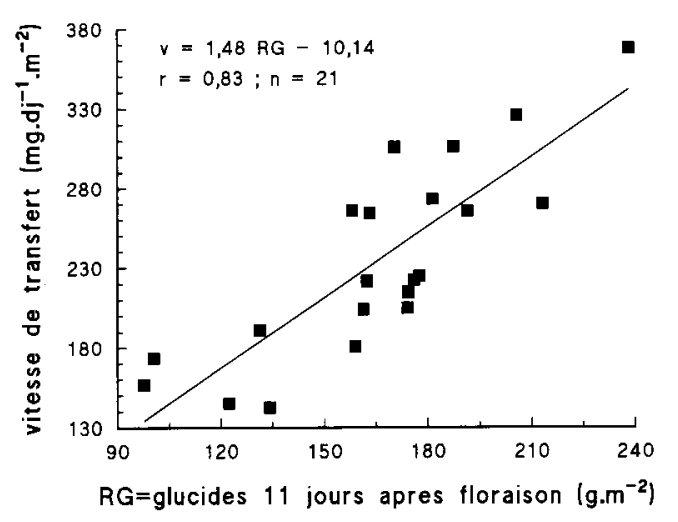

Fig 3. Effet du niveau des réserves glucidiques ( $R G$ ) sur le flux carboné $(v)$ depuis la tige. lée à la fois avec $p(r=0,61)$, avec NGE ( $r=$ $0,85)$ et avec le rendement $(r=0,63)$.

\section{Réserves carbonées à la floraison et les composantes du rendement}

Aucune influence des réserves glucidiques n'a été mise en évidence sur le nombre de grains exprimé par $\mathrm{m}^{2}$ ou par épi.

En revanche, les niveaux de réserves glucidiques par tige sont corrélés au rendement par épi $(r=0,57)$. Ces réserves sont corrélées aussi avec la vitesse de remplissage du grain $(r=$ 0,48 ) et avec le poids d'un grain $p(r=0,58)$ (fig 4). La corrélation avec la vitesse de remplissage est meilleure si l'on considère les réserves par grain $(r=0,67)$. La contribution au rendement des réserves carbonées, présentes à la floraison, est de $15 \%$ (tableau III). Calculée à partir de la quantité de réserves maximales, à environ $11 \mathrm{j}$ après la floraison, la contribution des réserves mobilisées (différence entre la floraison $+11 \mathrm{j}$ et la récolte) au poids sec du grain est de $20 \%$, soit le quart $(25,1)$ des glucides totaux du grain estimés à $82 \%$ du poids sec du grain (tableau III).

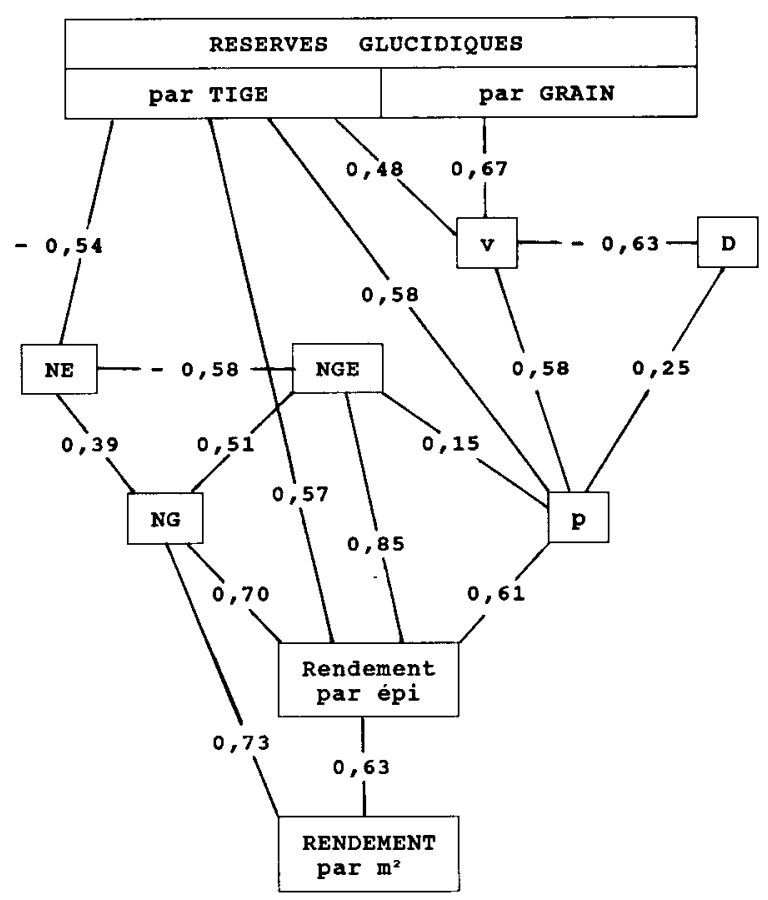

Fig 4. Les coefficients de corrélation entre différentes composantes du rendement chez 21 génotypes de blé tendre. ( $v$ et $D=$ vitesse et durée de remplissage du grain; $P=$ poids d'un grain; $N E=$ nombre d'épis $/ \mathrm{m}^{2} ; N G E=$ nombre de grains/épi; $N G=$ nombre de grains $/ \mathrm{m}^{2}, r=0,43$; coefficient de corrélation significatif pour $n=21$ et $\alpha=0,05$. 
Tableau II. Moyenne* des composantes du rendement des 21 génotypes.

\begin{tabular}{lrrrrrrr}
\hline & $N E$ & $N G E$ & $N G$ & $\begin{array}{c}V \\
\mu g^{\circ} \mathrm{C} . j\end{array}$ & $\begin{array}{c}D \\
{ }^{\circ} \mathrm{C} . j\end{array}$ & $\begin{array}{c}p \\
m g\end{array}$ & $\begin{array}{c}R D T \\
g / m^{2}\end{array}$ \\
\hline & & & & & & & \\
Moyenne & 736 & 28,1 & 20482 & 65,0 & 528 & 34,1 & 694 \\
Erreur sdt & 22 & 0,8 & 555 & 1,4 & 11 & 0,6 & 16 \\
Minimum & 570 & 21 & 14763 & 48,4 & 458 & 28,6 & 583 \\
Maximum & 937 & 35 & 25054 & 74,5 & 652 & 40 & 871 \\
\hline
\end{tabular}

$D$ : durée de remplissage; $V:$ vitesse de remplissage par grain; $p:$ poids du grain; NGE : nombre de grains par épi; NE : nombre d'épis par $\mathrm{m}^{2} ; R D T$ : rendement par $\mathrm{m}^{2} ; N G$ : nombre de grains par $\mathrm{m}^{2} ;{ }^{*}$ : moyenne calculée sur 4 prélèvement postfloraison.

Tableau III. Contribution des réserves glucidiques ( $R G$ ) au poids du grain.

\begin{tabular}{|c|c|c|c|c|c|}
\hline & \multicolumn{3}{|c|}{$\begin{array}{l}\text { Réserves glucidiques } \\
\text { à la floraison }\end{array}$} & $\begin{array}{l}\text { Contribution } \\
\text { RG floraison } \\
\text { \% RDT }\end{array}$ & $\begin{array}{c}\text { au rendement } \\
\text { RG mobilisées * } \\
\% \text { GT }\end{array}$ \\
\hline Moyenne & 101,6 & 140,0 & 5,0 & 14,7 & 25,1 \\
\hline Erreur sdt & 4,2 & 6,7 & 0,2 & 0,6 & 1,3 \\
\hline Minimum & 65,4 & 77,7 & 3,2 & 10,3 & 16,3 \\
\hline Maximum & 134,6 & 217,9 & 7,2 & 19,0 & 36,4 \\
\hline
\end{tabular}

* (réserves $11 \mathrm{j}$ après floraison - réserves à la récolte) / glucides totaux (GT) du grain, estimés à $82 \%$ du poids sec.

\section{DISCUSSION}

\section{Niveaux et dynamique des réserves $C$ et $N$}

Si on admet que l'assimilation nette et la répartition du $\mathrm{C}$ entre les parties aérienne et racinaire sont faiblement différenciées génétiquement il résulte que les principaux facteurs de variation de la quantité de $C$ mis en réserve sont la structure du peuplement (population d'épis par $\mathrm{m}^{2}$ ) et la durée de la période d'accumulation, dépendant de la date de la floraison.

Nos données confirment ces hypothèses; un retard de $1 \mathrm{j}$ à la floraison augmente la quantité des réserves glucidiques d'environ $4 \mathrm{~g} / \mathrm{m}^{2}$, ce qui représente $20 \%$ d'une croissance aérienne évaluée à $20 \mathrm{~g} / \mathrm{m}^{2}$ de matière sèche totale (résultat non présenté).

Notons aussi qu'un décalage dans le temps de la période d'accumulation des réserves implique une variation des conditions climatiques de cette période, avec des répercussions sur le niveau des réserves $C$ et sur la structure du peuplement, notamment sur la fertilité de l'épi (Fischer, 1985). Ainsi, un très fort retard de la floraison placerait la période d'accumulation des réserves dans des conditions climatiques défavorables (températures élevées, sécheresse...) et diminuerait ainsi la quantité de $\mathrm{C}$ mis en réserve. C'est le cas des génotypes Master et Arminda, les plus tardifs de notre expérimentation qui possèdent un niveau de réserves glucidiques plus faible que les génotypes plus précoces, Epiroux et Belaviso (tableau IV).

En ce qui concerne les réserves azotées, l'évolution différente selon le mode d'expression, en concentration (\%) ou en valeur absolue (mg/ tige) est due à l'augmentation du poids de la tige sous l'effet de l'accumulation des réserves glucidiques. En effet, la stabilité des quantités d'azote pendant les premiers j suivant la floraison atteste d'un maintien des équipements enzymatiques de la plante permettant une photosynthèse normale et une mise en réserve des glucides tant que les besoins pour la croissance 
Tableau IV. Proportion des glucides de réserve chez 4 génotypes de blé, 11 j après floraison.

\begin{tabular}{|c|c|c|c|c|}
\hline & Arminda & Master & Epiroux & Belaviso \\
\hline \multicolumn{5}{|l|}{ Glucides } \\
\hline$\%(\mathrm{MS})$ & 11,7 & 14,8 & 21,7 & 22 \\
\hline \multicolumn{5}{|c|}{ Proportions des différentes formes glucidiques } \\
\hline fructanes $D P>3$ & 0,6 & 6,5 & 15,7 & 9,8 \\
\hline kestose & 11,3 & 19,1 & 20,5 & 19 \\
\hline saccharose & 29,8 & 31,4 & 31,9 & 22 \\
\hline glucose & 34 & 22,2 & 13,3 & 23,3 \\
\hline fructose & 24,4 & 20,7 & 18,6 & 20 \\
\hline \multicolumn{5}{|c|}{ Flux glucidiques depuis la tige en mg de glucose } \\
\hline par $m^{2} .{ }^{\circ} \mathrm{C} . \mathrm{j}$ & 157 & 174 & 368 & 326 \\
\hline $\operatorname{par} \mathrm{g}^{\star} \cdot \mathrm{m}^{2} .{ }^{\circ} \mathrm{C} . j$ & 1,61 & 1,73 & 1,55 & 1,59 \\
\hline
\end{tabular}

* par gramme de glucides de réserve.

du grain ne sont pas trop importants. Ceci démontre l'utilité de l'expression de la teneur par rapport à la matière sèche structurale.

Pendant la phase linéaire de croissance du grain, a lieu une inversion de la balance production/utilisation d'assimilats qui est accompagnée par une mobilisation et un transfert des réserves $\mathrm{C}$ et $\mathrm{N}$.

Le flux de carbone exporté par $\mathrm{m}^{2}$ depuis les tiges (fig 2) est en moyenne, chez les 21 génotypes de $258 \mathrm{mg} \cdot \mathrm{m}^{-2} \cdot{ }^{\circ} \mathrm{C}^{-1} \mathrm{j}-1$. De plus, il est corrélé positivement avec le niveau maximal des réserves glucidiques mesurées $10 \mathrm{j}$ après la floraison. Une variation de $10 \mathrm{~g} \cdot \mathrm{m}^{-2}$ de ces réserves modifie le flux $\mathrm{C}$ d'environ $15 \mathrm{mg} \cdot \mathrm{m}^{-2} \cdot{ }^{\circ} \mathrm{C}^{-1} \cdot \mathrm{j}^{-1}$ (fig 3). L'effet positif de la quantité de réserves peut s'expliquer en grande partie par la concentration en substrat. Dans notre expérimentation, la concentration maximale en glucides, enregistrée environ $10 \mathrm{j}$ après floraison, varie de 11 à $26 \%$ selon le génotype. Elle est corrélée à la vitesse d'exportation, $(r=$ 0,$64 ; n=20$ ) mettant ainsi en évidence l'effet de la concentration de substrat sur sa vitesse d'hydrolyse et par suite de transfert (fig 3). Cet effet quantitatif pourrait être accompagné par un effet qualitatif, représenté par la forme chimique du substrat. Par exemple, avec l'augmentation de la concentration en glucides, la proportion des polymères de $D P>3$ augmente (Ollier et Triboi, 1989) : les génotypes Belaviso et Epiroux à forte teneur en réserves glucidiques $(\approx 22 \%)$ présen- tent une proportion plus importante de polymères supérieures à DP 3 que Master ou Arminda (tableau IV), qui pourrait modifier la cinétique d'hydrolyse. L'aspect détaillé concernant l'effet génotypique sur la composition des réserves glucidiques n'est pas présenté dans cet article. En terme de relation source-puits, l'effet positif de la quantité des réserves carbonées sur le flux d'exportation pourrait indiquer une limitation de type «source». Ceci est en concordance avec nos données antérieures qui montrent une relation positive entre la vitesse de remplissage du grain et la quantité des réserves (Triboi et al, 1985b).

En ce qui concerne l'utilisation des réserves azotées, notons le rôle important de la température car le flux moyen d'exportation depuis les tiges est de $6,7 \mathrm{mg} .{ }^{\circ} \mathrm{C}^{-1} \cdot \mathrm{j}^{-1} \cdot \mathrm{m}^{-2}$ (fig 2). Ce flux est spécifique de la population de 21 génotypes, caractérisée par une réserve moyenne de $6,9 \mathrm{~g}$ $\mathrm{N} / \mathrm{m}^{2}$ et une densité moyenne de 703 épis $/ \mathrm{m}^{2}$ (tableau I). Rapporté par tige le flux $\mathrm{N}$ est de $9,53 \mu \mathrm{g} \mathrm{N} .{ }^{\circ} \mathrm{C}^{-1}$. $\mathrm{j}^{-1}$ ou $0,191 \mathrm{mg} \mathrm{N}$ par jour pour une température moyenne journalière de $20^{\circ} \mathrm{C}$. Cette valeur est identique à celle obtenue par Simpson et al (1983), en expérimentant avec une seule variété cultivée en pots et en conditions contrôlées : $0,193 \mathrm{mg}$ pour les tiges, 0,192 $\mathrm{mg}$ pour les glumes et $0,202 \mathrm{mg}$ pour la feuille de l'épi. Si cette identité des valeurs est réelle et non fortuite, il en résulterait que le transfert de l'azote depuis un organe est contrôlé essentielle- 
ment par un même processus. II est certain que l'activité protéolytique jouerait un rôle important comme le soulignent Dalling et al (1976), mais les mécanismes de son contrôle restent encore à élucider. La vitesse de décharge du système vasculaire ou la force du puits pourrait intervenir; cependant, le facteur essentiel du contrôle de l'activité semble être la température (Triboi AM, 1984).

Enfin le rapport $\mathrm{C} / \mathrm{N}$ dans le flux d'assimilats transféré depuis les tiges, calculé d'après le rapport des 2 vitesses de transfert (flux $C=257 / 2,5$ $=103 \mathrm{mg} \mathrm{C}$; flux $\mathrm{N}=6,7 \mathrm{mg}$ ) est de 15,4 donc très proche de $\mathrm{C} / \mathrm{N}$ du grain. En considérant que le rapport $\mathrm{C} / \mathrm{N}$ des acides aminés transférés est de 4 , il résulte qu'environ $27 \%$ du $C$ sont transférés sous cette forme et $73 \%$ sous forme de glucides solubles ou autres.

\section{Rôle des réserves dans l'élaboration du rendement}

L'analyse des relations entre les différentes composantes du rendement a confirmé le rôle important du nombre de grains par $\mathrm{m}^{2}$ (fig 4). Cependant, un même NG peut être obtenu avec différents types de peuplement caractérisés par des structures (nombre de tiges, surface des feuilles, etc) et des fonctionnements différents (tableau V). Si un NG élevé est obtenu grâce à une population épis très élevée, le niveau des réserves $C$ par tige sera faible et la baisse de la vitesse du remplissage du grain induite par un NG élevé ne pourrait pas être compensée, d'où un poids de grain faible. Si un NG élevé est obtenu grâce à la fertilité de l'épi ce qui implique un NE faible, le niveau des réserves par tige sera élevé et la baisse de la vitesse sera compensée; le poids d'un grain sera stable. En effet, l'augmentation de la durée de remplissage induite par la baisse du flux ( $v$ ) expose le génotype à l'action des différents facteurs défavorables, d'ordre climatique, nutritionnel, métabolique, etc, qui n'autorisent que difficilement la réalisation du potentiel de poids d'un grain.

Les résultats présentés mettent ainsi en évidence le rôle de la composante rendement par épi (RDTE) conjointement au nombre de grains par $\mathrm{m}^{2}$ (NG) dans le déterminisme du rendement.

À priori, RDTE pourrait représenter un critère d'adaptation au milieu ou de stabilité car il bénéficie de l'effet favorable d'un fort niveau de réserves carbonées par tige. En revanche, NG re-
Tableau V. Schéma hypothétique d'élaboration du rendement chez 2 peuplements de blé produisant par des voies différentes un même nombre de grains par $\mathrm{m}^{2}$.

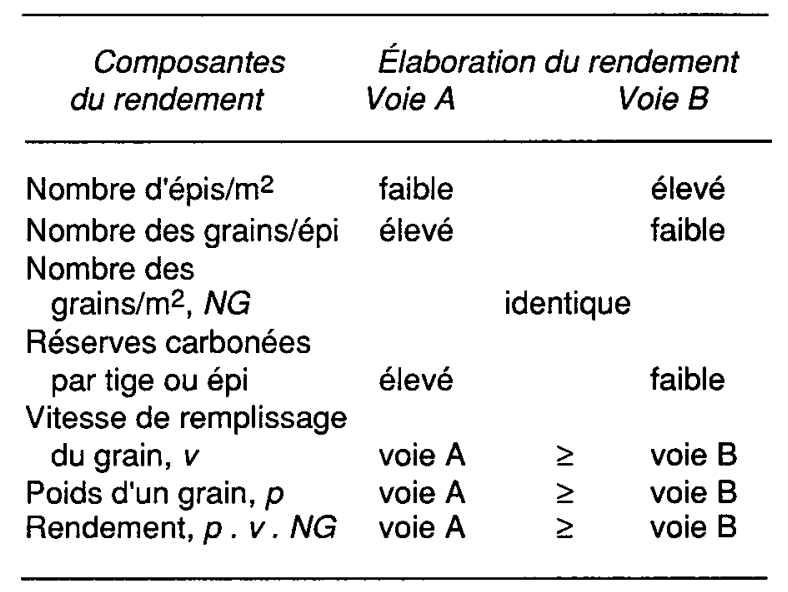

présenterait plutôt un «potentiel génétique» qui ne pourrait s'exprimer qu'un l'absence des facteurs limitants du milieu.

En ce qui concerne le rôle des réserves azotées plusieurs voies d'actions sont possibles. Selon l'origine de l'azote prélevé (tiges ou/et feuilles), ces réserves pourraient agir d'une part sur la durée de fonctionnement (Spiertz et Vos, 1984) et d'autre part sur la photosynthèse et donc sur la disponibilité en carbone. Nos données ne permettent de mettre en évidence aucune de ces voies.

Soulignons aussi qu'une augmentation des réserves $\mathrm{N}$ pourrait accroître le flux de $\mathrm{N}$ vers le grain sans changer la durée de remplissage (Triboi et Triboi, 1989). Des études complémentaires sont donc nécessaires pour répondre à ces questions.

Enfin, en dehors des implications possibles dans le processus de sélection, ces résultats peuvent orienter le choix des génotypes et des techniques culturales adaptées au milieu.

\section{RÉFÉRENCES}

Austin RB, Ford MA, Edrich JA, Blackwell RD (1977) The nitrogen economy of winter wheat. J Agric Sci 88, 159-167

Austin RB, Morgan CL, Ford HA, Blackwell RD (1980) Contribution to grain yield from pre-anthesis assimilation in tall and dwarf barley phenotypes in two contrasting seasons. Ann Bot 45, 309-319

Blaclow WM, Darbyshire B, Pheloung P (1984) Fructans polymerised and depolymerised in the internodes of winter wheat as grain filling progressed. Plant Sci Lett 36, 213-218 
Dalling MJ, Boland G, Wilson JH (1976) Relation between acid proteinase activity and redistribution of nitrogen during grain development in wheat. Aust $J$ Plant Physiol 3, 721-730

Fischer RA (1985) Number of kernels in wheat crops and the influence of solar radiation and temperature. J Agric Sci 105, 446-461

Judel GK, Mengel K (1982) Effect of shacking on nonstructural carbohydrate and their turnover in culms and leaves during the grain filling period of spring wheat. Crop Sci 22, 958-962

Kuhbauch W, Thome V (1989) Nonstructural carbohydrate of wheat stems as influenced by sink-source manipulation. J Plant Physiol 134, 243-250

Ollier JL, Triboi E (1989) Étude qualitative des réserves carbonées chez 21 génotypes et modélisation quantitative de la biosynthèse des fructanes. Colloque Ecophysiologie, Versailles 25-26 Mars

Pollock CJ, Jones T (1979) Seasonal patterns of fructan metabolism in forage grasses. New Phytol 83, 9-15

Simmons RS (1987) Growth development and physiology. In: Wheat and wheat improvement. Agronomy Monograph 13, 2nd edn, ASA-CSSA-SSSA, 1-97
Simpson RJ, Lambers H, Dalling MJ (1983) Nitrogen redistribution during grain growth in wheat (Triticum aestivum L). Plant Physiol 71, 7-14

Spiertz JHJ, Vos J (1984) Grain growth of wheat and its limitation by carbohydrate and nitrogen supply. In: Wheat modelling (Day W Atkin RK eds) Plenum Press, New York, 129-143

Triboi AM (1984) Métabolisme azoté dans la plante. Trait d'Union Agric 89, 16-20

Triboi E (1986) Maîtrise du rendement quantitatif et qualitatif chez le blé. Colloque franco-roumain “Rendement des Céréales», INRA Versailles, 296323

Triboi E (1990) Modèle d'élaboration du poids du grain chez le blé tendre. Agronomie 10, 191-200

Triboi E, Triboi AM (1989) Flux, quantité et origine de l'azote absorbé par le blé. Colloque “Ecophysiologie du blé», Versailles 25-26 mai

Triboi E, Blanchon J, Magne J (1985a) Déterminisme du poids moyen du grain chez le blé. Effet sur la variation du rendement. CR Séances Acad Agric Fr, séance du 20 mai, 71, 871-886

Triboi E, Rousset M, Lemercier E (1985b) Élaboration du poids du grain. Écophysiologie du blé CR 1984 , INRA, $67-100$ 